\title{
Comorbidity and its Impact on Patients with COVID-19
}

\author{
Adekunle Sanyaolu $^{1}$ (1D $\cdot$ Chuku Okorie $^{2} \cdot$ Aleksandra Marinkovic $^{3} \cdot$ Risha Patidar $^{3} \cdot$ Kokab Younis $^{4} \cdot$ Priyank Desai $^{5}$. \\ Zaheeda Hosein $^{6} \cdot$ Inderbir Padda ${ }^{7} \cdot$ Jasmine Mangat $^{6} \cdot$ Mohsin Altaf $^{8}$
}

Accepted: 12 June 2020 / Published online: 25 June 2020

(C) Springer Nature Switzerland AG 2020

\begin{abstract}
A novel human coronavirus, severe acute respiratory syndrome coronavirus 2 (SARS-CoV-2), was identified in Wuhan, China, in December 2019. Since then, the virus has made its way across the globe to affect over 180 countries. SARS-CoV-2 has infected humans in all age groups, of all ethnicities, both males and females while spreading through communities at an alarming rate. Given the nature of this virus, there is much still to be learned; however, we know that the clinical manifestations range from a common cold to more severe diseases such as bronchitis, pneumonia, severe acute respiratory distress syndrome (ARDS), multi-organ failure, and even death. It is believed that COVID-19, in those with underlying health conditions or comorbidities, has an increasingly rapid and severe progression, often leading to death. This paper examined the comorbid conditions, the progression of the disease, and mortality rates in patients of all ages, infected with the ongoing COVID-19 disease. An electronic literature review search was performed, and applicable data was then collected from peer-reviewed articles published from January to April 20, 2020. From what is known at the moment, patients with COVID-19 disease who have comorbidities, such as hypertension or diabetes mellitus, are more likely to develop a more severe course and progression of the disease. Furthermore, older patients, especially those 65 years old and above who have comorbidities and are infected, have an increased admission rate into the intensive care unit (ICU) and mortality from the COVID-19 disease. Patients with comorbidities should take all necessary precautions to avoid getting infected with SARS CoV-2, as they usually have the worst prognosis.
\end{abstract}

Keywords Coronavirus $\cdot$ COVID-19 $\cdot$ SARS-CoV-2 $\cdot$ Clinical features $\cdot$ Comorbidity $\cdot$ Diabetes $\cdot$ Hypertension

This article is part of the Topical Collection on Covid-19

Adekunle Sanyaolu

sanyakunle@hotmail.com

1 Federal Ministry of Health, Abuja, Nigeria

2 Essex County College, Newark, NJ, USA

3 Saint James School of Medicine, The Quarter, BWI, Anguilla

4 Mount Royal University, Calgary, Alberta, Canada

5 American University of Saint Vincent School of Medicine, Kingstown, Saint Vincent and the Grenadines

6 Caribbean Medical University School of Medicine, Willemstad, Curaçao

7 University of Washington, Seattle, WA, USA

8 Xavier University School of Medicine, Oranjestad, Aruba

\section{Introduction}

Coronavirus disease (COVID-19) caused by severe acute respiratory syndrome coronavirus 2 (SARS-CoV-2) is a global pandemic that initially started in Wuhan, China, and spread extremely quickly, making its way to over 180 countries. As the novel coronavirus continues to evolve, there are still many limitations to our knowledge of who exactly this virus would impact critically. Older adults and people of any age who have underlying medical conditions, such as hypertension and diabetes, have shown worse prognosis [1]. Diabetic patients have increased morbidity and mortality rates and have been linked to more hospitalization and intensive care unit (ICU) admissions [1]. People with chronic obstructive pulmonary disease (COPD) or any respiratory illnesses are also at higher risk for severe illness from COVID-19 [2]. The risk of contracting COVID-19 in patients with COPD is found to be 4-fold higher than patients without COPD [2]. 
A retrospective study of middle-aged and elderly patients with COVID-19 found that the elderly population is more susceptible to this illness and is more likely to be admitted to the ICU with a higher mortality rate [3]. The age-related changes in the geriatric population may be due to the changes in lung anatomy and muscle atrophy which results in changes in physiologic function, reduction of lung reserve, reduction of airway clearance, and reduction of the defense barrier function [3].

The clinical outcomes and length of stay directly correlate with the underlying conditions and age of the COVID19 patient. Researchers in China studied 344 patients in the ICU with COVID-19. Of the 344 subjects, 133 patients died on the 28th day with a median survival of 25 days [4]. Of these patients, there were many with different comorbidities with 141 patients having hypertension [4]. In survivors, the median duration of hospitalization for a negative test result was 12 days [4]. Furthermore, another study conducted in China studied 633 COVID-19 patients, of whom 247 patients had at least one comorbidity and that concluded that older individuals, greater than 60 years, were more likely to exhibit a more severe form of COVID-19 disease [5]. During the study, 25 patients with a median age of 69.3 years died giving an effective mortality rate of $3.77 \%$ [5].

It has been concluded that the older the patients are, the more likely they are to succumb to the COVID-19 disease. In the USA, the Centers for Disease Control and Prevention (CDC) uses COVID-NET in 14 states to monitor the demographics of COVID-19 patients who are being hospitalized [6]. From March 1 through 30,2020 , there were a total of 180 patients on COVIDNET, of which $89.3 \%$ of the patients had an underlying comorbidity [6]. Of the 180 patients, $94.4 \%$ aged 65 years and older had at least comorbidity [6]. The most common comorbidities found were obesity, hypertension, and diabetes mellitus [6]. The purpose of this paper is to review these comorbidities, given that most patients with severe COVID-19 cases had comorbidity. Furthermore, we would like to examine specific comorbidities in relation to the COVID-19 disease progression and outcomes based on the literature report since the outbreak.

\section{Methodology}

An electronic literature search was performed using PubMed, Google Scholar, EBSCOhost, Mendeley, and MedLine Plus. The search was limited to peer-reviewed articles published from January to April 20, 2020. An article was selected if it included keywords such as coronavirus, COVID-19, SARS-CoV-2, clinical features, comorbidity, diabetes, and hypertension. Articles were then reviewed and included based on the applicability to the topic.

\section{Presence of Comorbidities and the Clinical Characteristics and Outcomes of COVID-19}

\section{Clinical Characteristics}

Confirmed and reported cases of COVID-19 have a wide range of symptoms from mild complaints, such as fever and cough, to more critical cases associated with difficulty in breathing [7]. Some of the most common symptoms include cough, fever, chills, shortness of breath (SOB), muscle aches, sore throat, unexplained loss of taste or smell, diarrhea, and headache [8]. Symptoms can start as mild and become more intense over 5 to 7 days, worsening if pneumonia develops in patients [8]. Approximately, 1 out of 6 infected individuals become seriously ill and develop difficulty in breathing, especially in the elderly with underlying health conditions [9].

A meta-analysis study of COVID-19 patients, as depicted in Fig. 1, showed fever (88.8\%) as the most common symptom, followed by dry cough (68\%) and fatigue (33\%) [10]. Other symptoms noted were productive cough (28.5\%), SOB $(17 \%)$, muscle pain $(14.4 \%)$, sore throat $(11.4 \%)$, and headache $(10.2 \%)$ [10]. The least common symptoms were diarrhea $(4.4 \%)$, nausea and vomiting $(4.1 \%)$, rhinorrhea $(3.2 \%)$, abdominal pain $(0.16 \%)$, and chest pain $(0.11 \%)$ [10].

Symptoms of COVID-19 may appear anytime from 2 to 14 days after exposure; therefore, 14-day quarantine is recommended [7]. The average incubation period for COVID-19 is approximately 5.2 days [11]. In Wuhan, China, the most common symptoms observed from the onset of this outbreak include fever, cough, and fatigue, while some features that were not so prevalent were sputum production, headache, hemoptysis, and gastrointestinal (GI) symptom such as diarrhea [12, 13]. Another study that was conducted in Beijing, China, showed that the average age of patients with COVID-19 was 35.5 years and had a mean of 3.5 days from the onset of symptoms to admission in the hospital [14]. The same study also showed that fever was present in $87.5 \%$ of patients, which persisted for 6.5 days, and symptoms resolved 2.5 days after a negative test result [14].

Some individuals who are infected do not develop any symptoms at all, and about $80 \%$ of positive cases recover from the disease without any treatment [9]. However, there have also been instances of transmissions of COVID-19 from one person to another before the person became aware of being sick, or the symptoms were so mild that the person did not know he/she had the illness [15]. It becomes essential to seek medical attention immediately if a person suspects that he/she may have been 
Fig. 1 Clinical characteristics of COVID-19 from January 24 through March 16, 2020. Data obtained from Research Square, a meta-analysis of the 2019 novel coronavirus, showing clinical characteristics observed in patients, as of April 8, 2020 [10]
COVID-19 Clinical Characteristics (Jan. 24, 2020 -

Mar. 16, 2020)

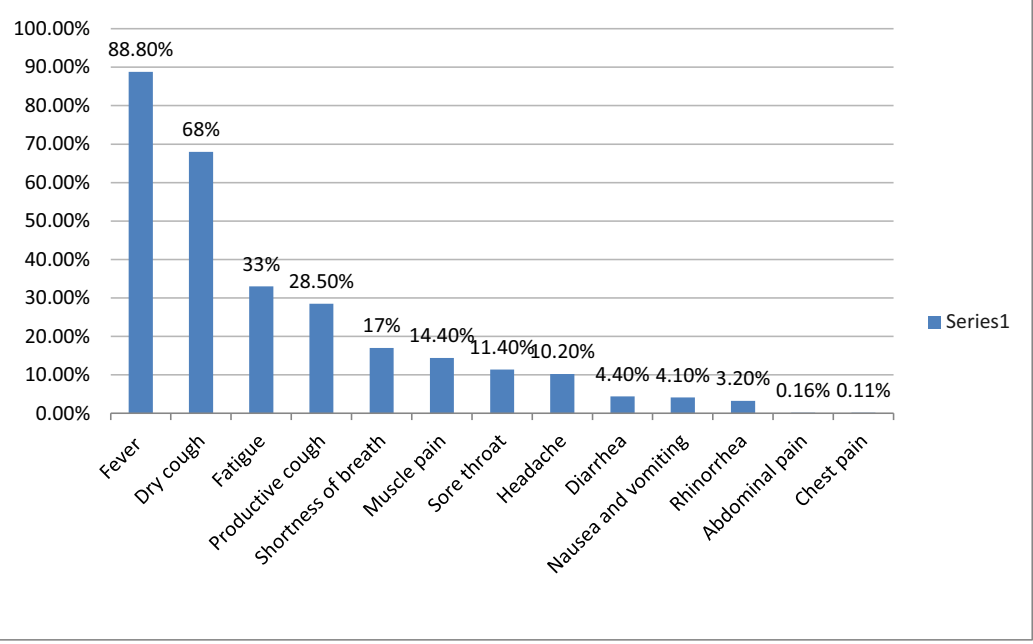

Note: Data obtained from Research Square, a meta-analysis of the 2019 novel coronavirus, showing clinical characteristics observed in patients, as of April 8, $2020^{10}$. infected or is a confirmed case of COVID-19 experiencing respiratory distress, has blue lips, is in constant pain, or has pressure in the chest [7]. Also, it is important to note that COVID-19 is more infectious than SARS-CoV and MERS-CoV due to its numerous epidemiological and biological characteristics [16].

\section{Comorbidities}

Due to COVID-19 being a relatively new and understudied disease, the data available is limited. However, from the cases that emerged, it was observed that comorbidities increase the chances of infection [7]. Based on current information and clinical expertise, the elderly, especially those in long-term care facilities, and people of any age with serious underlying medical conditions are at a greater risk of getting COVID-19 [7]. The elderly, a vulnerable population, with chronic health conditions such as diabetes and cardiovascular or lung disease are not only at a higher risk of developing severe illness but are also at an increased risk of death if they become ill [15]. People with underlying uncontrolled medical conditions such as diabetes; hypertension; lung, liver, and kidney disease; cancer patients on chemotherapy; smokers; transplant recipients; and patients taking steroids chronically are at increased risk of COVID-19 infection [7].

A meta-analysis study on COVID-19 comorbidities, as depicted in Fig. 2, had a total of 1786 patients, of which 1044 were male and 742 were female with a mean age of 41 years old [10]. The most common comorbidities identified in these patients were hypertension (15.8\%), cardiovascular and cerebrovascular conditions (11.7\%), and diabetes $(9.4 \%)[10,17]$. The less common comorbidities were coexisting infection with HIV and hepatitis B $(1.5 \%)$, malignancy $(1.5 \%)$, respiratory illnesses $(1.4 \%)$, renal disorders $(0.8 \%)$, and immunodeficiencies $(0.01 \%)$ [10].

Patients with moderate to severe asthma are at a disadvantage because this virus affects their respiratory tracts, leading to increased asthmatic attacks, pneumonia, and acute respiratory distress [7]. According to the CDC's morbidity and mortality weekly report, $34.6 \%$ of patients aged 18 to 49 years old have an underlying chronic lung disease, such as asthma [6]. In Iran, a case study reported that genetic predisposition to COVID-19 can increase the probabilities of getting infected with SARS-CoV-2 and can lead to death from this virus [18]. This genetic predisposition was seen in 3 brothers in Iran who were 6 years apart in age (54, 60, and 66) years who died due to COVID-19, despite no identified comorbidities and living separately from one another [18]. As of January 2, 2020, Wuhan, China, had 41 patients admitted to the hospital who tested positive for COVID-19, of which $73 \%$ (30/41) were men with a median age of 49 years old, $66 \%$ (27/41) of these patients had been exposed to the Huanan market, 32\% (13/41) had underlying diseases such as diabetes $20 \%(8 / 41)$, hypertension $15 \%$ (6/41), and cardiovascular $15 \%(6 / 41)$ [12]. Patients who are HIV positive, along with a low CD4 count and not on antiretroviral therapy, have a higher risk and incidence of other medical issues including COVID-19 [7]. According to the International Aids Society (IAS), people 
Fig. 2 Comorbidities associated with COVID-19 infection from January 24 through March 16, 2020. Data obtained from Research Square, a meta-analysis of the 2019 novel coronavirus, showing clinical comorbidities observed in patients, as of April 8, $2020[10]$

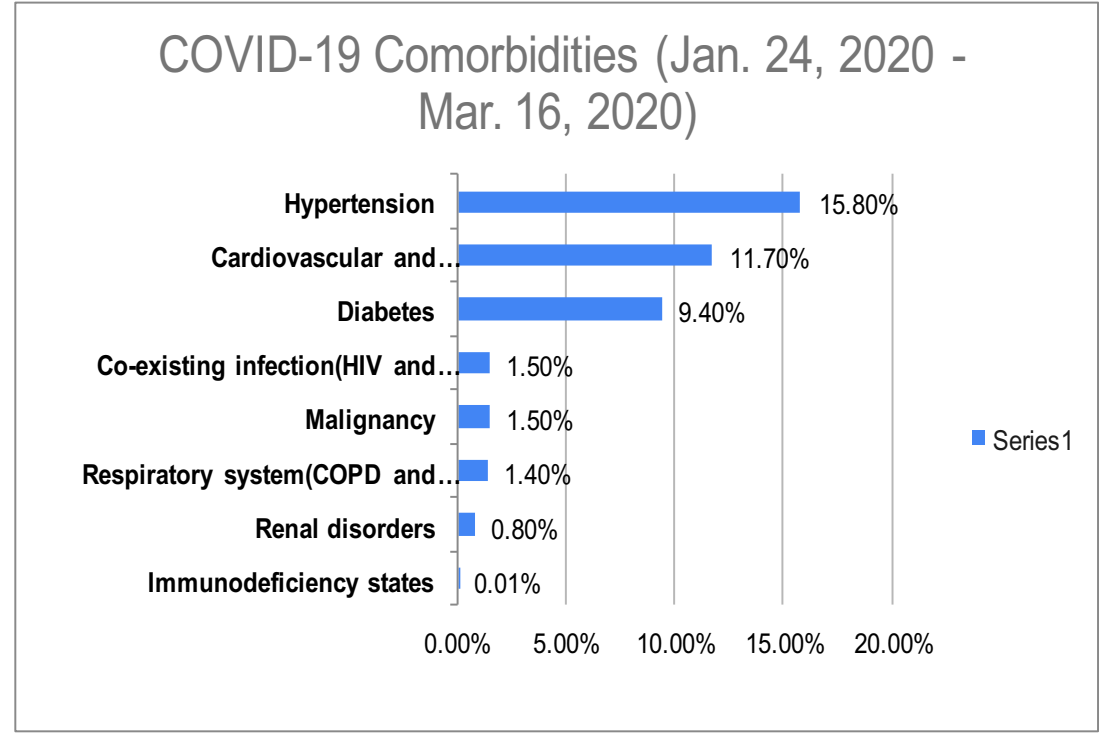

Note: Data obtained from Research Square, a meta-analysis of the 2019 novel coronavirus, showing clinical comorbidities observed in patients, as of April 8, $2020^{10}$. who are human immunodeficiency virus (HIV) positive, taking antiretroviral treatment, and do not have a low CD4 count will have the same risk of having COVID-19 as one without HIV [19, 20].

As depicted in Fig. 3, a population-based surveillance report via COVID-19-Associated Hospitalization Surveillance Network (COVID-NET) reported clinical data on 1478 COVID-19-positive patient hospitalizations from March 1 to March 30, 2020 [6]. Among the 1478 patients studied, 12\% of adults showed clinical data of underlying medical conditions with the most prevalent being hypertension (49.7\%) and obesity a close second $(48.3 \%)$ [6]. Other medical conditions included chronic lung disease $(34.6 \%)$, diabetes mellitus (28.3\%), and cardiovascular diseases (27.8\%) [6].

\section{Outcome}

COVID-19 can cause severe disease leading to hospitalization in ICU and potentially death, especially in the elderly with comorbidities [21]. According to the CDC, 8 out of 10 deaths reported in the USA occurred in adults 65 years old and above [7]. Roughly $80 \%$ of COVID-19-positive cases result in full recovery from the illness without any hospitalizations or interventions [9]. There are still many facts that we do not know about COVID-19 due to gaps in knowledge; therefore, many studies are underway to better understand this virus [15].

Wuhan, China, had 41 patients admitted to the hospital who tested positive for COVID-19 from December 16, 2019 , to January 2, 2020, and the clinical outcomes are shown
Fig. 3 COVID-19 underlying medical conditions in confirmed hospitalized cases from March 1 through March 30, 2020. Data obtained from the CDC showing the hospitalization rates and characteristics of patients hospitalized with laboratoryconfirmed coronavirus disease 2019 , during the period of March 1-30, 2020, and depicted here as of April 17, 2020 [6]

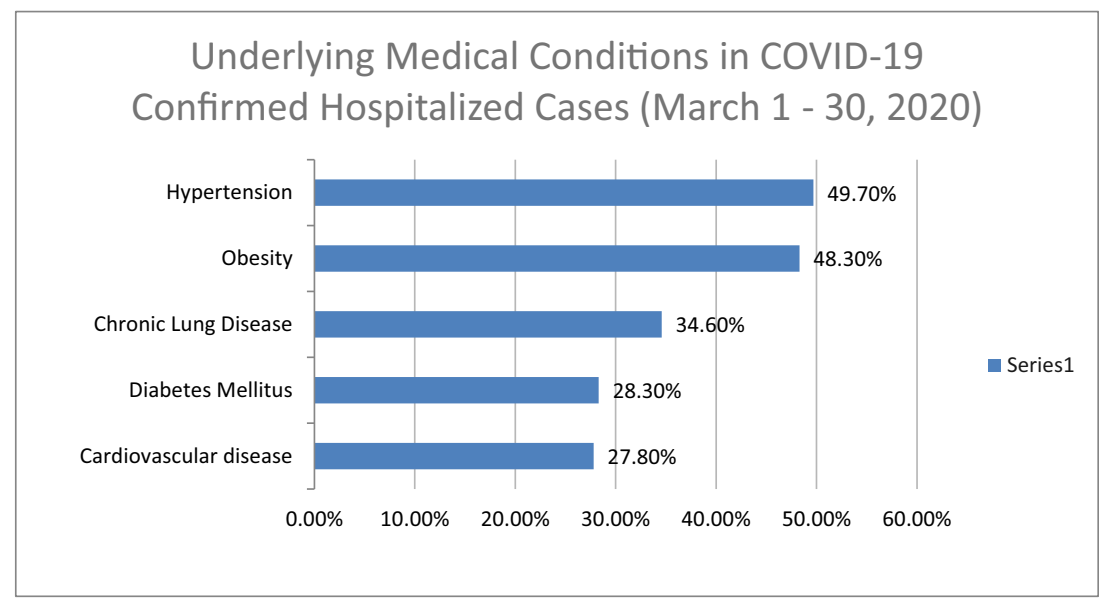

Note: Data obtained from the CDC showing the hospitalization rates and characteristics of patients hospitalized with laboratory-confirmed coronavirus disease 2019, during the period of March 1-30, 2020 and depicted here as of April 17, $2020^{6}$. 
Fig. 4 Clinical outcomes of COVID-19 from December 16 through January 2, 2020. Data obtained from the Lancet showing clinical features of patients infected with 2019 novel coronavirus in Wuhan, China, as of January 24,2020 [12]

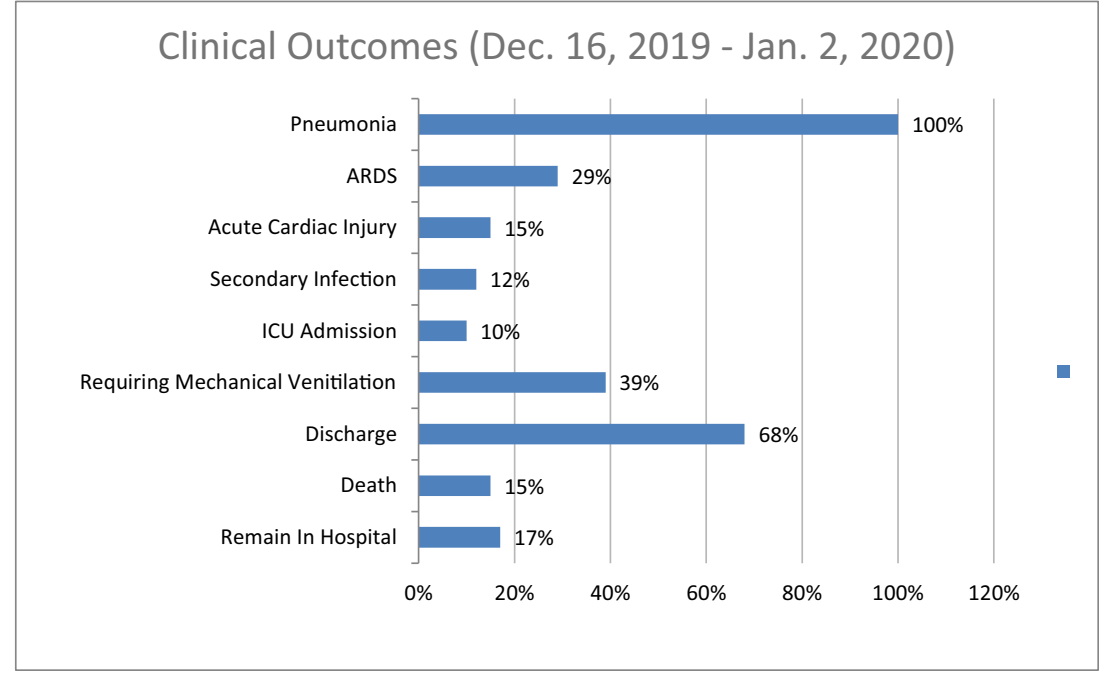

Note: Data obtained from the Lancet showing clinical features of patients infected with 2019 novel coronavirus in Wuhan, China, as of January $24,2020{ }^{12}$. in Fig. 4 [12]. Of the 41 patients with confirmed COVID-19 in the hospital, 100\% had pneumonia, 29\% developed acute respiratory distress syndrome (ARDS), $15 \%$ exhibited an acute cardiac injury, and $12 \%$ acquired a secondary infection [12]. The majority of these patients (68\%) were discharged, $17 \%$ remained hospitalized, $10 \%$ ended up in the ICU, and $15 \%$ ended in death [12].

From February 20 to March 5, 2020, a study was conducted in Evergreen, Washington, on the characteristics and outcomes of 21 critically ill patients with confirmed COVID-19 diagnosis [22]. This study showed that $71 \%$ of these patients required mechanical ventilation and acute respiratory arrest was seen in all of them [22]. Within $72 \mathrm{~h}$ of developing ARDS, 53\% of these patients showed increasing severity of respiratory problems, leading to poor short-term outcomes and a higher risk of death [22]. As of March 17, 2020, the mortality rate was $67 \%$ in these patients, with $24 \%$ critically ill and $9.5 \%$ discharged from hospital [22].

As shown in the CDC's mortality and morbidity weekly report in Fig. 5, case mortality increased as the patient's age increased [21, 23]. Among the age group $\leq 19$ years, no ICU admission or mortality was reported [21]. COVID-19 has also been noticed in children, but the disease took a more moderate course when compared with adults. The prognosis was also seemingly better, and deaths were infrequent [24].

On April 29, 2020, WHO reported over 3 million confirmed COVID-19 cases worldwide, with over 200,000 of these cases resulting in mortality [25]. The majority of these
Fig. 5 COVID-19

hospitalizations, ICU admissions, and deaths from February 12 through March 16, 2020. Data obtained from the CDC showing severe outcomes among patients with coronavirus disease 2019 (COVID-19) in the USA from February 12 through March 16, 2020 , and reported, as of March 27, 2020 [21]

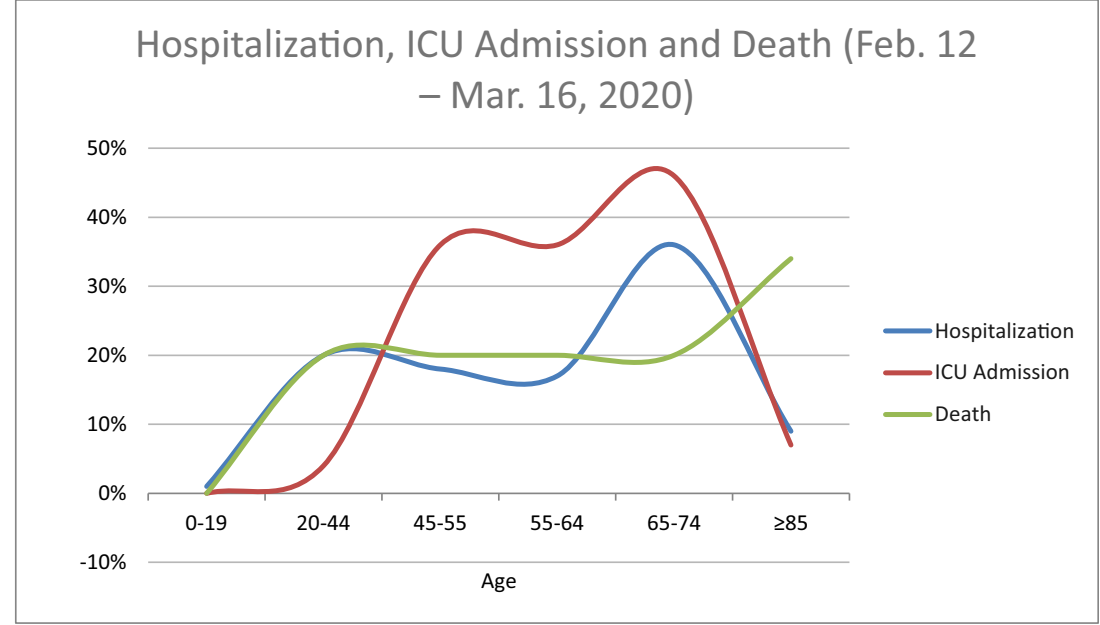

Note: Data obtained from the CDC showing severe outcomes among patients with coronavirus disease 2019 (COVID-19) in the USA from February 12, 2020 through March 16, 2020 and reported, as of March 27, $2020^{21}$. 
Table 1 Leading comorbidities among COVID-19 deaths in NY, USA

\begin{tabular}{ll}
\hline Comorbidities & Death $\%$ \\
\hline Hypertension & $55.4 \%$ \\
Diabetes & $37.3 \%$ \\
Hyperlipidemia & $18.5 \%$ \\
Coronary artery disease & $12.4 \%$ \\
Renal disease & $11.0 \%$ \\
Dementia & $9.1 \%$ \\
COPD & $8.3 \%$ \\
Cancer & $8.1 \%$ \\
Atrial fibrillation & $7.1 \%$ \\
Heart failure & $7.1 \%$ \\
\hline
\end{tabular}

Data reported by hospitals, nursing homes, and other health facilities to the New York State Department of Health, as of April 6, 2020 [26]

deaths are within the USA with over 50,000, followed by Italy with over 25,000 and Spain with over 20,000 [25]. New York is being hit the hardest by the novel coronavirus spreading across the USA, with more cases and deaths per capita than any other state. Table 1 reports that over $86 \%$ of COVID-19 deaths involved at least one comorbidity, according to the New York State Department of Health [26].

\section{Discussion}

Multiple comorbidities are associated with the severity of COVID-19 disease progression. Many of the poorer outcomes for COVID-19 have been related to cardiovascular comorbid conditions [27]. However, this may be a direct result of the cardiovascular condition itself or attributed to other comorbidities along with a cardiovascular condition [27]. Patients with type 2 diabetes were also more likely to have increased severity of COVID-19 [28]. In a cohort study of 7337 patients with COVID-19 with and without type 2 diabetes, it was shown that those with type 2 diabetes required increased interventions for their hospital stay versus those that were nondiabetic [28]. It was shown that those with poorer blood glucose control had an all-around increased mortality rate than those with better glucose control [28]. The study concluded that patients with blood glucose specifically those in the range of 3.9 to $10.0 \mathrm{mmol} / \mathrm{L}$ had overall decreased composite adverse events and mortality [28]. It was noted that poor blood glucose control resulted in a substantially increased risk of complications and death [28]. Among other comorbidities, chronic obstructive pulmonary disease (COPD) has also been associated with poor disease progression. A meta-analysis of multiple studies in China found that there was a four-fold increase in mortality in patients with preexisting COPD that were diagnosed with
COVID-19 [29]. In this analysis, the smoking status of patients and severity of COVID-19 were also studied. Only one study was found to have an association with smoking and severe course of COVID-19 [29]. After this study was removed, there was no significant association with smoking and severe COVID-19 disease [29].

Patients with acute respiratory distress syndrome and respiratory failure associated with the novel coronavirus may be linked to a prothrombotic coagulopathy [30]. The autopsy of COVID-19-related deaths revealed dispersed microthrombi in the pulmonary vasculature, demonstrating an occlusive etiology of respiratory failure [30]. A 38 to $100 \%$ improvement was noted in three cases with COVID-19-related acute respiratory distress syndrome (ARDS) and respiratory failure, following treatment with intravenous (IV) antithrombotic alteplase [30]. The results, although reassuring, were transient in two of the three cases with recovery succumbing posttreatment [30]. The use of anti-thrombolytic therapy needs to be further studied to ascertain that the results are due to treatment and not chance alone [30]. A retrospective meta-analysis including 1558 COVID-19 patients was conducted until March 1, 2020, on subjects with underlying comorbidities and the risk for acquiring COVID-19 [31]. Common comorbidities such as hypertension, COPD, diabetes, and cardiocerebrovascular disease were observed to be the more significant risk factors in subjects when compared with other underlying disease states [31]. Other than intense supportive measures, a definitive treatment has yet to be identified [31]. Authorities speculate that subjects with comorbidities were linked to a more severe disease outcome when infected with the novel coronavirus when compared with patients with no underlying disease [31]. The study was met with barriers due to the diminutive sample size and some subjects having more than one underlying comorbidity [31]. In literature and earlier research, underlying diseases increase the mortality rate in patients who acquired COVID-19 with cardiac disease and diabetes being the most significant risk factors [32]. Once again, insufficient sample size and inadequate time have led to limitations in the data collected [32].

\section{Conclusion}

Over 180 countries have been affected by COVID-19, resulting in mass death worldwide. As cases evolve globally, it has been noted that persons with underlying chronic illnesses are more likely to contract the virus and become severely ill.

Due to SARS CoV-2 being a relatively new virus, the data available is limited. However, patients with comorbidities have more deteriorating outcomes compared with patients without. COVID-19 patients with history of hypertension, obesity, chronic lung disease, diabetes, and cardiovascular 
disease have the worst prognosis and most often end up with deteriorating outcomes such as ARDS and pneumonia. Also, elderly patients in long-term care facilities, chronic kidney disease patients, and cancer patients are not only at risk for contracting the virus, but there is a significantly increased risk of death among these groups of patients.

Of the cases reported worldwide, symptoms of COVID-19 ranged from mild respiratory disease to severe illness, which requires intubation and mechanical ventilation. Since there is a period where the patient is asymptomatic, and the incubation period is between 2 and 14 days, it is difficult to establish an early diagnosis, much less curb the transmission of the infection if the patient is unknowingly exposed. However, within this period, if respiratory symptoms develop, it is important to seek immediate care.

Therefore, patients with comorbidities should take all necessary precautions to avoid getting infected with SARS CoV2 , as they usually have the worst prognosis. These precautions include regular handwashing with soap and water or use of alcohol-based hand sanitizer, limiting person-to-person contact and practicing social distancing, wearing a face mask in public places, and overall limiting going to public areas at this time unless it is necessary. Hence, there is a need for a global public health campaign to raise awareness, on reducing the burden of these comorbidity illnesses causing deaths in COVID-19-infected patients.

Authors' Contribution A.S., conceptualization and design and approval of the final version, responsibility for the accuracy, and integrity of all aspects of research. C.O., supervision and revising the article for intellectual content and editing. A.M., drafting the article and editing and project administration. R.P., drafting the article and acquisition of data. K.Y., drafting the article and acquisition of data. P.D., drafting the article and acquisition of data. Z.H., drafting the article and acquisition of data. I.P., data analysis and interpretation of data. J.M., data analysis and interpretation of data. M.A., data analysis and interpretation of data.

\section{Compliance with Ethical Standards}

Conflict of Interest The authors declare that they have no conflict of interest.

\section{References}

1. Singh AK, Gupta R, Ghosh A, Misra A. Diabetes in COVID-19: prevalence, pathophysiology, prognosis, and practical considerations. Diabetes Metab Syndr Clin Res Rev. 2020;14(4):303-10 [Accessed April 18, 2020, https://reader.elsevier.com/reader/sd/pii/

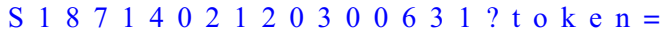
9053C7A9DEA8F9AD35620DE8A76E569E800C744D2785BF 1F3E13C425A07B78B480145B9E04D94BE41439983005F17A52].

2. Zhao Q, Meng M, Kumar R, Wu Y, Huang J, et al. The impact of COPD and smoking history on the severity of COVID-19: a systemic review and meta-analysis. J Med Virol. 2020. https://doi.org/ 10.1002/jmv.25889 [Accessed April 18, 2020, https://www.ncbi. nlm.nih.gov/pubmed/32293753].
3. Liu K, Chen Y, Lin R, Han K. Clinical features of COVID-19 in elderly patients: a comparison with young and middle-aged patients. J Inf Secur. 2020;15(30):1-5 [Accessed April 18, 2020 https://www.journalofinfection.com/article/S0163-4453(20)30116$\mathrm{X} / \mathrm{pdf}]$.

4. Wang Y, Lu X, Chen H, Chen T, Su N, et al. Clinical course and outcomes of 344 intensive care patients with COVID-19. AJRCCM. 2020;201:1430-4 [Accessed April 20, 2020, https:// www.atsjournals.org/doi/pdf/10.1164/rccm.202003-0736LE].

5. Zhang J, Wang X, Jia X, Li J, Hu K, et al. Risk factors for disease severity, unimprovement, and mortality of COVID-19 patients in Wuhan, China. Clin Microbiol Infect. 2020. [Accessed April 20, 2020;26:767-72. https://doi.org/10.1016/j.cmi.2020.04.012.

6. Garg S, Kim L, Whitaker M, O'Halloran A, Cummings C, et al. Hospitalization rates and characteristics of patients hospitalized with laboratory-confirmed coronavirus disease 2019 - COVID-NET, 14 states, March 1-30, 2020. Centers for Disease Control and Prevention: MMWR. 2020;69(15):458-64 [Accessed April 18, 2020, https://www.cdc.gov/mmwr/volumes/69/wr/mm6915e3.htm].

7. CDC. Coronavirus (COVID-19): symptoms of coronavirus. Centers for Disease Control and Prevention. 2020. [Accessed April 18, 2020, https://www.cdc.gov/coronavirus/2019-ncov/ symptoms-testing/symptoms.html].

8. Maragakis LL. Coronavirus symptoms: frequently asked questions. Johns Hopkins Medicine. 2020; [Accessed April 18, 2020, https:// www.hopkinsmedicine.org/health/conditions-and-diseases/ coronavirus/coronavirus-symptoms-frequently-asked-questions].

9. WHO. Q\&A on coronaviruses (COVID-19). World Health Organization. 2020. [Accessed April 18, 2020, https://www.who. $\mathrm{int} /$ news-room/q-a-detail/q-a-coronaviruses].

10. Paudel SS. A meta-analysis of 2019 novel coronavirus patient clinical characteristics and comorbidities. Research Square. 2020. https://doi.org/10.21203/rs.3.rs-21831/v1 [Accessed April 18, 2020, https://www.researchsquare.com/article/rs-21831/v1].

11. Li Q, Med M, Guan X, Wu P, Wang X, et al. Early transmission dynamics in Wuhan, China, of novel coronavirus-infected pneumonia. N Engl J Med. 2020;382:1199-207. https://doi.org/10. 1056/NEJMoa2001316 [Accessed April 18, 2020, https://www. nejm.org/doi/full/10.1056/NEJMoa2001316].

12. Huang C, Wang Y, Li X, Ren L, Zhao J, et al. Clinical features of patients infected with 2019 novel coronavirus in Wuhan, China. Lancet. 2020;395(10223):497-506. https://doi.org/10.1016/S01406736(20)30183-5 [Accessed April 18, 2020 https://www.thelancet. com/journals/lancet/article/PIIS0140-6736(20)30183-5/fulltext].

13. Yang J, Zheng Y, Gou X, Pu K, Chen Z, Guo Q, et al. Prevalence of comorbidities in the novel Wuhan coronavirus (COVID-19) infection: a systematic review and meta-analysis. Int $\mathrm{J}$ Infect Dis. 2020;S1201-9712(20):30136-3. https://doi.org/10.1016/j.ijid. 2020.03.017 [Accessed April 20, 2020, https://pubmed.ncbi.nlm. nih.gov/32173574/?from_term=covid+cormorbidities\&from_pos= $1 \&$ from_exact_term $=$ covid+comorbidities].

14. Chang $\bar{D}$, Mo G, Yuan X, Tao Y, Peng X, Wang FS, et al. Time kinetics of viral clearance and resolution of symptoms in novel coronavirus infection. AJRCCM. 2020;201:1150-2. https://doi.org/10. 1164/rccm.202003-0524LE. [Accessed April 18, 2020, https://www. atsjournals.org/doi/pdf/10.1164/rccm.202003-0524LE].

15. BCCDC. COVID-19 vulnerable populations. British Columbia Centre for Disease Control. 2020. [Accessed April 18, 2020, http://www.bccdc.ca/health-info/diseases-conditions/covid-19/ vulnerable-populations].

16. Meo SA, Alhowikan AM, Al-Khlaiwi T, Meo IM, Halepoto DM, et al. Novel coronavirus 2019-nCoV: prevalence, biological and clinical characteristics comparison with SARS-CoV and MERSCoV. Eur Rev Med Pharmacol Sci. 2020;24(4):2012-9. https:// doi.org/10.26355/eurrev_202002_20379 [Accessed April 20, 2020, https://pubmed.ncbi.nlm.nih.gov/32141570/]. 
17. Zhou F, Yu T, Du R, Fan G, Liu F, et al. Clinical course and risk factors for mortality of adult inpatients with COVID-19 in Wuhan, China: a retrospective cohort study. Lancet. 2020;395(10229): 1054-62. https://doi.org/10.1016/S0140-6736(20)30566-3 [Accessed April 20, $2020 \mathrm{https} / /$ www.thelancet.com/pdfs/ journals/lancet/PIIS0140-6736(20)30566-3.pdf].

18. Yousefzadegan S, Rezaei N. Case report: death due to novel coronavirus disease (COVID-19) in three brothers. Am J Trop Med Hyg. 2020;102:1203-4. https://doi.org/10.4269/ajtmh.20-0240. [Accessed April 18, 2020, https://www.ajtmh.org/content/ journals/10.4269/ajtmh.20-0240].

19. IAS. COVID-19 and HIV: what you need to know. The International AIDS Society. 2020. [Accessed April 20, 2020, https://www.iasociety.org/covid-19-hiv].

20. WHO. Q\&A on COVID-19, HIV, and antiretrovirals. World Health Organization. 2020. [Accessed April 20, 2020, https://www.who. $\mathrm{int} /$ news-room/q-a-detail/q-a-on-covid-19-hiv-and-antiretrovirals].

21. CDC. Severe outcomes among patients with coronavirus disease 2019 (COVID-19) - United States, February 12-March 16, 2020. Centers for Disease Control and Prevention: MMWR. 2020;69(12):343-6 [Accessed April 18, 2020, https://www.cdc. gov $/ \mathrm{mmwr} /$ volumes $/ 69 / \mathrm{wr} / \mathrm{mm} 6912 \mathrm{e} 2 . \mathrm{htm}$ ?s cid=mm6912e $2 \mathrm{w}]$.

22. Arentz M, Yim E, Klaff L, Lokhandwala S, Riedo FX, Chong M, et al. Characteristics and outcomes of 21 critically ill patients with COVID-19 in Washington state. JAMA. 2020;323:1612. https:// doi.org/10.1001/jama.2020.4326. [Accessed April 19, 2020, https://jamanetwork.com/journals/jama/fullarticle/2763485].

23. Wu C, Chen X, Cai Y, Xia J, Zhou Z, et al. Risk factors associated with acute respiratory distress syndrome and death in patients with coronavirus disease 2019 pneumonia in Wuhan, China. JAMA Intern Med. 2020:e200994. https://doi.org/10.1001/ jamainternmed.2020.0994 [Accessed April 20, 2020, https:// jamanetwork.com/journals/jamainternalmedicine/fullarticle/ 2763184].

24. Ludvigsson JF. Systematic review of COVID-19 in children shows milder cases and a better prognosis than adults. Acta Paediatr. 2020;109:1088-95. https://doi.org/10.1111/apa.15270. [Accessed April 20, 2020, https://onlinelibrary.wiley.com/doi/full/10.1111/ apa.15270].
25. WHO. Coronavirus COVID-19. World Health Organization. 2020. [Accessed April 29, 2020, https://covid19.who.int/].

26. Franki R. Comorbidities the rule in New York's COVID-19 deaths. Hospitalist. 2020; [Accessed June 1, 2020, https://www.thehospitalist.org/hospitalist/article/220457/coronavirus-updates/ comorbidities-rule-new-yorks-covid-19-deaths].

27. W-Jie G, Liang W-H, He J-X, Zhong N-S. Cardiovascular comorbidity and its impact on patients with COVID-19. Eur Respir J. 2020;55:2001227. https://doi.org/10.1183/13993003.01227-2020. [Accessed May 30, 2020, https://erj.ersjournals.com/content/early/ 2020/04/20/13993003.01227-2020].

28. Zhu L, She Z-G, Cheng X, Qin J-J, Zhang X-J, et al. Association of blood glucose control and outcomes in patients with COVID-19 and pre-existing type 2 diabetes. Cell Metab. 2020; [Accessed May 30, 2020, https://www.sciencedirect.com/science/article/pii/ S1550413120302382].

29. Zhao Q, Meng M, Kumar R, Wu Y, Huang J, et al. The impact of COPD and smoking history on the severity of COVID-19: A systemic review and meta-analysis. J Med Virol. 2020. https://doi.org/ 10.1002/jmv.25889 [Accessed May 30, 2020, https://pubmed.ncbi. nlm.nih.gov/32293753/].

30. Wang J, Hajizadeh N, Moore EE, McIntyre RC, Moore PK, et al. Tissue plasminogen activator (tPA) treatment for COVID-19 associated acute respiratory distress syndrome (ARDS): a case series. J Thromb Haemost. 2020. https://doi.org/10.1111/jth.14828 [Accessed May 30, 2020, https://onlinelibrary.wiley.com/doi/full/ 10.1111/jth.14828].

31. Wang B, Li R, Lu Z, Huang Y. Does comorbidity increase the risk of patients with COVID-19: Evidence from meta-analysis. Aging (Albany NY). 2020;12(7):6049-57. https://doi.org/10.18632/ aging.103000 [Accessed May 30, 2020, https://pubmed.ncbi.nlm. nih.gov/32267833/].

32. Li B, Yang J, Zhao F, Zhi L, Wang X, et al. Prevalence and impact of cardiovascular metabolic diseases on COVID-19 in China. Clin Res Cardiol. 2020;109:531-8 [Accessed May 30, 2020, https://link. springer.com/article/10.1007/s00392-020-01626-9].

Publisher's Note Springer Nature remains neutral with regard to jurisdictional claims in published maps and institutional affiliations. 